\title{
Variety of Medical Discourses and their Impact Upon Narratives of Illness in West Sumatera
}

\author{
Jiri Majer ${ }^{1 凶}$ \\ ${ }^{1}$ Univerzita Komenskeho, Slovakia
}

DOI: http://dx.doi.org/10.15294/komunitas.v5i2.2732

Received : September 2012; Accepted: August 2013; Published: September 2013

\begin{abstract}
The aim of this article is to give concrete examples of how narratives influence the way person speaks about and experience his illness. The research was conducted in West Sumatera where people know a diverse range of health discourses: western medicine, local ethno-medicine or Islamic way of treatment called ruqyah. This study is part of my research project, which focused on the process of creation and negotiation of a diagnosis within a multicultural and multi-discursive context in Indonesia. This study concludes that people in West Sumatera withdraw from several medical discourses to give meaning to both their health and illness.
\end{abstract}

Keywords: cross-cultural relativism; discourse; negotiation of disease; narratives of illness.

\section{INTRODUCTION}

I have been thinking a lot about the question of how spirit possession differs from mental disorders. A person normally explains the unknown through concepts which are familiar to him/her. I was introduced to spirit possession in Indonesia just few days after my arrival. I am glad that I had a change to witness ritual of exorcism right at the beginning of my stay. I immediately faced the dilemma if I should try to enforce my point of view and insist on calling ambulance because what I witnessed is an epileptic seizure or I should leave my ethnocentric interpretation of this trance and body spasm and accept the existence of the phenomenon of spirit possession (quotation from my ethnographic notes diaryy).

The inspiration for my research in the region of West Sumatra and this article was a vast presence of spirit possession in West Sumatera. I assume that this spirit possession cause by demon, jinnee or other entity, which are the consequence of cultures st- rongly bounded with mono/polytheistic religion and/or animism. It is due to the fact, that they describe/create in their cosmologies very different entities which are able to cause the possession. In the case of West Sumatra it is first of all Islam, which brings jinnee in its cosmology.

Though we can find spirit possession phenomenon in the other parts of world; the spirit possession in West Sumatra is in many aspects unique. Even though the local culture of Minangkabau is closely linked with Islam it is still matrilineal and partly matriarchic society. In this cultural context, one can be a witness how Islamic religious way of treatment - called ruqyah - is combining with local ethno-medicine and scientific findings of biomedicine are in clash with the traditional way of curing.

In one geographic area, there are several discourse fields in which and between whose people move with their individuality and personal strategy while being confronted with environment. These fields are 
overlapping each other. It contributes to specific consequences that person can understand his/her condition - which seen through the local cultural lances is exceeding the culturally determinated concept of normality - both symptoms of the spirit possession or particular diseases which are anchored in the discourse of biomedicine. Subsequently individual can choose one method of treatment or try to combine them. In extreme it means, that in doctors' waiting rooms one can meet a person who connects their condition with supernatural entities or opposite; in the narration of somebody who searched a help at a traditional healer, we can detected narration which are based on the biomedicine(Barnard, 1996).

Hence, in this diversity, I had a chance to focus on the process of negotiation about final nature of particular disease and influence of prescribing diagnosis on individuals narration. In this article I deal with a question of relativity of abnormality and influence of particular discourse on the individuals' utterances. Thus I discuss how one's narration is influenced and/or confronted with wider frames of references, which prescribe meanings to subjective interpretation of illness.

\section{RESEARCH METHOD}

The unit of research subject were older than 18 years and their origins were in area of West Sumatra. The interviews were conducted both, with patients of local asylum; and individuals who search for help at ruqyah or dukun. In addition there were interview with the doctors (psychiatrist and psychologists). For the compact palette, I led interviews with representatives of local ethno-medicine (dukun) and ruqyah. Even though it is possible to assume that there will be various utterance among different generations and genders, my research sample did not reflect these nuances. My research donot focus on the comparison of diversity across the population. My research rather aimed on the analysis of previously described topic on more general level.

For this research and due to its nature I chose the qualitative method of the data gathering, through participant observation and semi structured questionnaires. The core of this questions set, which was focused on several area of the research's interest, served both the interviewer as a leash for guidance of the interviews; and the respondents for directing their narrations. As well, it allowed interviewer to focus on interesting and outstanding details which appeared during the interviews and in give a more freedom to navigate their utterance. The actual questions, which were divided into several categories, were connected with concepts which were crucial for this research because they could be linked with the main areas of the interest of my work.

\section{DISCUSSION}

In this paper I completely avoid any inclination to medicalizate the phenomenon of spirit possession. I focus on socio-cultural context which is permeated by several discourses, which prescribe specific meanings to particular phenomena. Therefore I use three diagnoses from biomedicine and one which comes from local ethno-medicine and ruqyah:

1) Epilepsy will be in this paper understood as a specific neurologic disease. There are several types of it, which manifest different symptoms. For epilepsy which affects the whole brain, the is characteristic seizure, is. muscle spasm and unconsciousness. Patient does not (usually) remember these seizures and after it is gone one need some time to get back to normal.

2) Schizophrenia will be understood as mental disease which is manifested by changes in the process of thinking and mood disorder. It is exhibited mostly by hallucinations, paranoia, disillusion or confused speeches or train of thoughts. This disease is connected with strong social dysfunction.

3) Dissociative disorders have assumed psychogenic origination. They are linked with traumatic incident, problems which patient is not able to solve, or with a disbalanced social relationship. In the 
context of biomedicine these disorder are typical by separation of particular psychical content from the rest of consciousness (Práško et al. 2007).

4) Spirit Possession in the area of West $\mathrm{Su}$ matra can be distinguished in two types based on the local ethno-medicine and religious discourse. One can be introduced to ,acute“ by other words direct spirit possession, when after the body of a person is accommodated external entity, the person faints and one's body is suffering from a spasms. The other case can be interpreted as a "long term". The external entity which is present in a person manifests itself gradually and the level of power can have increasing or decreasing tendency. In this case a possessed person might (or not at all) sense the presence of the entity and reflect it, or one might fall under its dominion.

These particular health disorders are described on very general level. It is not caused by my incapability to specify them, hence, bring concrete definitions, which would be anchored in appropriate discourses. "Vagueness" of their conceptualization is result of my tendency to describe them on very "objective" level. Even though the diseases which I work with - e.g. epilepsy, schizophrenia and dissociative disorders are possible to specify within biomedical discourse, for my research and this article crucial are the interpretation of their external manifestation - symptoms - by general language. I am not concerned about the reflection on discourse definition, but rather about their social references. The particular health seeker or his/her social surrounding which decide about the following steps in choosing the way of treatment, is usually not provided with the expert knowledge of the present discourses. Therefore I assume it is more useful to work with diagnoses which are described on the general level.

\section{Constructivism and Relativity of abnor- mality}

In every society and its culture it is particular kind of medicine and/or cosmology of religion which changes one's perception on surrounding world and perceiving of an illness. Whereas uninitiated child sees a neutral manifestation of particular condition of disharmony, culturally biased person interprets his/her condition as a specific labeled disease based on its symptoms. Therefore it is in every society, a medical/religious discourse, "creates" particular nature of disease and the way of its diagnostic and treatment. With this process is connected the aspect, that the observer transmit into the object of observation even his/her relationship forward this object (Bourdieu, 1998; Holland, 2004).

In the context of constructivist theories, the approach to the question of relativity of abnormality is obvious. Interpretation and diagnostic of "madness" is culturally relative. The anthropology theorists detect culturally bound syndromes and their manifestations (Littlewood 1996); and described historical changes within biomedical discourse (Foucault 1994, 2007). Hence in this work, which is dealing with various kind of medicine, it is necessary to reflect even the relativity of abnormality on its own. Based on the constructivism, it is crucial to emphasize, that both, the biomedical knowledge and diagnoses are social constructed (Sudnow 1967) and thus they are discursively determinated. It is necessary to perceive the diagnosis as a process which is being created by the signified and signifying, while it results in creation of particular disease. "The final relation between signifying and signified is on every level of medical experience redistributed between the symptoms which are signifying and the disease which is signified, between description and object which is described, between a situation and the prognosis, between disorder and the feeling which indicated it " (Foucault 1994: XIX).

Important part during the process of negotiation of the final disease's nature is enacted by the representatives of particular discourses. It is them, who based on socially accepted expert knowledge, are capable to identify (thus diagnose) some abnormality, but at the same time - based on the power 
relation - can apply these constructs of disease on the afflicted person. Additionally the doctor or dukun/ ruqyah accepts just these symptoms which fits into his/her initial framework and the patient or possessed person become a passive recipient of the dictate of particular discourse and its representatives.

In the context of different ways of treatments, patients are examinated and their diseases are diagnosed on the principles of preexisting scenarios, scripts and schemas. Phenomena which cannot be fit is refused, overlooked or forcibly changed in the particular way, so they can be transmitted into a discourse which is peculiar to the specific treatment. In the realm, it means that if doctors who are linked with biomedicine face a phenomenon of a spirit possession, they will transform received information into the chemical and physical symptoms of some misbalanced conditions within a body; and psychiatrists into diagnoses of psychiatry. Similarly dukun or ruqyah will perceive a mental disorder. On this procedure it is possible to reflect the process of creation of a diagnosis, which needs to be understood as a specific cultural perspective, which creates what the disease is and how it should be treated (Fabrega in Helman 1978: 134).

While considering biomedicine which has been established in the area of West Sumatra, it is necessary to reflect both different socio-cultural framework; and the role of language on its own. It is due to the fact which Wartowsky point out. If we want to create a framework for understanding relation between disease and illness we have to deepen our knowledge about the way how psychosocial and cultural factors influence the disease. Hence, disease must be understood as socio-cultural and historical phenomenon (Wartowsky in Good 1977), which is anchored in the particular language. This is linked with the very core of this article. The final negotiated diagnoses from discourse of biomedicine, ethno medicine or ruqyah are - and must be considered as - remarkably different. In the context of mentioned relativity it is important to reflect as well that particular sensation of a body can be seen by individual from western society as traumatic or a symptom of a diseases, while by person from different socio-cultural framework it can be understood as a kind of punishment or symptom of spiritual experience (von Peter 2008). Therefore, different interpretation of surrounding world obviously bias the understanding of an event, which is/is not perceived as traumatic (Kohrt and Hruschka 2010).

Further on, in the case of variously grasped causality we can point out problems of application of the concepts which are rooted in particular discourse. For example see Wikan, who demonstrates how some experience cannot be separated from intersubjective and public sphere (Wikan in von Peter 2008:644).

Hence, it is obvious, that the meanings of illness are socially constructed. Furthermore Good (1977) conclude: “The meaning of an illness is generated socially as it is used by individuals to articulate their experiences of conflict, thus becoming linked to typical syndromes in the society. The meanings of used terms change as the social conditions and the social context alter" (39-40). Later he argues that: "the expression and impression of illness is bounded with the interconnection of images, complex of symbols and feelings, which are deeply rooted in the structure of society and its culture. This final nature of an illness is constituted by the mean of its expression in the context of a social interaction. While person articulates subjective condition, he/she expects the meaningful reaction, which will ease the undesirable condition of the affected person" (Good 1977: 48 - 49). In the case of my analysis of the discursive construction of mental disorders, epilepsy or phenomenon of spirit possession, I align with opinion of von Peter, who suggests to leave the discussion about which diagnosis is better or more correct then the others. He emphasizes rather to focus on the experiences on they own and on the question how these conditions, which are understood as traumatizing or as a health disorders are created, experienced, embodied, expressed and treated (2008).

In other dimension the role of cultural frame and the interpretation of an illness 
are documented by Acharya a Norhcott. They focus on the definition of a culture as a moral medicine, which is presented by Indian migrant women in Canada (Acharya and Norhcott 2007: 630). If we involve religion in the concept of culture, we can detect similar tendency in the area of West Sumatra. That is because any religion provides its followers with a set of tool, practices which can cure them. The subsequent question is, if we can find a faith in curing function of various cultural practices even in so called western society and western medicine. Biomedicine admits placebo and its healing effect, which is connected with the patients' belief of its effectiveness. The success of placebo is based on the patients absence of knowledge, that the medication is ordinary substance, that is not - based on the biomedical discourse - capable of giving expected treatment. Besides, one can find a faith in the power of curing springs or air in the several areas.

A new perception on the socio-culturally anchored negotiation of a disease is presented by the cross-cultural perspective. Different frames of references, which are emphasized by this perspective allow us to both critically consider the human universality and the diseases; and underline the cultural bias of biomedicine which is based on particular concepts and values. The central argument within cross-cultural perspective is that: "The emphasis of research and analysis should thus be shifted away from questions of whether these disease entities are 'the same' or 'different' across cultures - or 'right' or 'wrong' in particular contexts - to that of how people make sense of life events (von Peter 2008: 647)". Hence it is obvious that diagnosed diseases cannot be constituted as natural entities which effect body or psyche. As Frake point out, they should be rather understood as social and historical realities. A diagnosis is transcription of patient's subjective condition into categories of diseases according to interpretation of symptoms which characterize particular disease (Frake in Good 1977).

While accepting the relativistic approach which is rooted in constructivist theo- ries I oppose the biological paradigm which assumes universality of reactions and experiences which are evoked by same health condition. Hence, this approach is as well bounded with the reflection of discourse and its impact on utterance of health seekers and their understanding of their health condition. But at this point, in addition, it is necessary to emphasize, that during any research or data analysis the social science's reductionism should be avoided. This kind of reductionism is connected with overlooking of what exactly respondents are saying or doing and replacing it with interpretation of social scientists, who are loading respondents' utterances with additional meanings (Latour 1999).

\section{The Impact of Discourse during a Nego- tiation of a Disease}

Analysis of conducted interviews was based on the theoretical frame of phenomenology, which assume that it is not possible to prescribe objectivity to lived and perceived reality. Hence the core of interest is the perception and interpretation of individuals. Therefore this theoretical approach is focused on subjective construction of objectified reality. In common experience in the case of interviews with health seekers, it was (usually) their subjective feeling of an illness or disharmony. Therefore their narration, which was bounded with this experience, mirrored (un)reflected changes in their conditions. While in the case of representatives of various discourses the offered help was the aspect which was in common to these narratives. Hence it was closely bounded with reflection of negotiated abnormality and particular curing procedures.

Before I bring concrete example of a young girl who visited ruqyah, it is important to mention, that in case of all narrations it is necessary to reflect their sociocultural context. It is crucial to consider that utterance of patient from mental clinic or health seeker who visited dukun or ruqyah as well as psychiatrist/psychologist or any representative of other discourses is biased by one of the symbolic or reference frames which are presented in the area of West Su- 
matra. Narrations are not mere output from dialogs. It is necessary to understand them as subjective interpretations situated in social and cultural context which shaped them (Kirmayer 2006). Hence, this point became very important during the analysis. It might be luring to compare or confront particular utterance with more objective description thus discourse. Slipping to such level of analysis might be caused by the fact, that all different discourses, claim attribute of "objective truth". Although they offer "genuineness" it is important to emphasize that any kind of medical discourse or religious practice is one of many institutionalized interpretation of somebody`s subjective condition. Therefore, for my research and following analysis, it was unfruitful to contemplate about what "really" happened, but it was better to focus on what particular respondent says. That means to reflect and analyze how he/she perceives his/her illness and what meaning he/she is giving to it.

Even though I leave a side the question of general validity in case of particular narratives about spirit possession or mental disorder, it is necessary to reflect its credibility. This aspect can be illustrated on the example of my interview with a girl, who together with her mother, told a story of her illness which supposed to represent a spirit possession. She came to an open space/garage which was the "ordination" of a ruqyah. As she came in she was asked to fill a form with several questions about her condition and mostly about her feeling while praying or reading The Quran. The fact that she had to fill this form revealed two important aspects about the practice of this ruqyah. (1) "The enrolment" of a patient and prechecking of patient by several written questions points out a change of the discourse of ruqyah which happened under the influence of western medicine, for which this way of pre-diagnosing of patient is very typical. (2) The way how the questions were formed pre-shaped the patients utterance. The narration was changed under the pressure of questions which guided his/her memory. They made a person choose particular worlds and accept concepts of disease and its syndromes which were anchored in the discourse of Islam. As the person is asked about presence of negative feeling while reading holy book of The Quran, he/she is already confronted with a discourse which is indicating crucial subjective feeling. If person tickles answer "yes", this one word determinates future reaction of ruqyah. He would pay higher attention to the girl's narration, because as he can read in advance, the result of the questionnaire reveals - without any single subjective interpretation of the patient - possible presence of a jinnee. On the other hand if a person chooses answer "no" for all or most of the questions the ruqyah, it does not expect a presence of the evil entity in one's body. Hence without the single spoken word with a patient, the diagnosis is/is not prescribed. At this point it is necessary to underline that it is not a body, a result of various biological, chemical or physical tests that are so typical for the western medicine, but it is a memory of a patient which was guided by the questionnaire that has the power to determinate the diagnosing process. Hence the past experience was put in words by the power of very specific questions. It is not any more the girl and her story of subjective feeling and experience of disharmony, illness. It is rather a testimony, reaction on the discourse and its methodology and classification.

As she spoke with a ruqyah who asked her several question about her condition, she had to search for words describing (hence changing) her subjective feeling of a disharmony in terms, concepts thus construct of religious discourse. When she was asked what is the matter with her, what she suffers from, she replied that she might be possessed. She chose a term that describes a situation when self of jinnee enter edbody and suppressed one's own self. Out of sudden her unspecified fluid experience and feelings of abnormality were carved into a term out of religious discourse. While she was negotiating nature of her disorder she had to cooperate and search for words, which "make sense" in the situation she was. She had to translate her subjective feeling in the language of religion. 
After this girl finished with the help of her mother answering all the question, she finally undergone a ritual of exorcism of which serves as both a tool for identification a spirit possession (proving a jinnee's presence in one's body) and the treatment of this condition. But her case jinnee, which is supposed to be embodied in her, did not react on the reading of The Quran. The expected jinnee's negative reaction which would be exhibited as a body agony did not happen. Hence in the context of ruqyah it means that she was not possessed. Following interpretation was obvious. Based on the absence of jinnee's reaction, which is significant condition of spirit possession, the presence of jinnee was not proved. Even though that applied method of treatment was not successful in treating her condition it did not adumbrate its malfunction. It rather pointed out, that this girl was suffering from other disease then spirit possession.

Hence on this story we can illustrate the attitude of any and every discourse, which claims the "true" of its explanatory and classificatory model, and methodological approach. Discourse is the universe of objective categories and prescribed treatment which is applied on individual's condition. Thus it is better to understand it as an objective model which is used while the patient's subjective illness is negotiated with the constructs of disorder that is labeled as particular disease. During this negotiation I witnessed a situation when discourse through its methodology of identification and categorization of disease/spirit possession applied/revealed - based on its power over an individual - the "true", hence reinterpreted the girl's condition as nonpossession so it questioned the credibility of her utterance. She learnt, forced by this discursive dictate, to accept the "fact" that she is not possessed. She had to reevaluate her subjective feelings of disorder in other frameworks and search for verifying response in other discourses.

Hence, this example clearly illustrates that while the diagnosis is negotiated within particular discourse, subjective feeling and narration is/might be perceived as inferior.
In such clashing situation different discursive frames offer new space, field of meanings for the health seekers to "retell" subjective experiences in retroactive reinterpretation. Thus, the story of this girl consequently reveals the process of reconstruction of understanding one's subjective experiencing and interpreting of unwilling health condition. The clash while negotiating particular diagnose, thus linking subjective feelings and interpretation with institutionalized and categorized meanings, which are valid for particular discourse, meant for this girl and her family, that they had to search for other frameworks of meanings or had to accept that her condition is not abnormal.

For complexity of this problemacy, it is necessary to mention that unwilling condition can be identified first by the social surrounding of an individual who does not have to reflect any changes in his or her condition. This situation was more common in the case of patients of mental clinic where I conducted my research. Significant in such examples is that the social surrounding was the active participant which recognized a change in one's behavior and acting, which was perceived as abnormal. Consequently the discourse of chosen medicine did or did not assign particular category of disease thus mental disorder - which supported or negated the "validity" of the assumption of social surrounding even without subjective feeling of presence of any illness.

For this discussion, it does not matter if an individual consciously experience a condition which he/she identify as abnormal, or if it is the social surrounding which assume presence of some concrete disease. Crucial is the fact that in every case there is process of searching of (new) meaning within particular discursive framework. As it was mentioned in the cultural context of Minangkabau these frameworks are presented by discourse of western medicine, local ethno-medicine and ruqyah. Each offer a meaning, prescribed diagnosis (1) which is given by the representative of particular discourse and or (2) which is accepted by the health seekers or is denied and individuals are trying to negotiate diagnosis from other 
discourse. Hence it is obvious that discourses determinate the way of perception of and individual and his/her social surrounding on one's health condition. For deeper analysis the question of social stigmatization cannot be left a side. While in the culture of Minangkabau the presence in asylum and assigned diagnosis of mental disorder leads to strong social exclusion, recognized spirit possession increase the social inclusion.

In the context of the discussion about the social stigma of diagnosed mental disorders, which at the culture of Minangkabau refer to deprecated "madness", it is appropriate to take into account what Csordas et al. mentioned about the narratives of involved individuals. "Narration does not refer only to individuals who reflect his/her particular subjective condition, but it is also bounded with a future activities and experiences ... Therefore, lived experiences and social activities have a complex relationship with the narrations on their own" (Csordas et al, 2010: 53). Hence, it clearly implies that individuals in the area of West Sumatra will try to negotiate the diagnoses, which will be partly assigned according to his/her narrative, not only based on one's feeling and experiencing of illness, but also according to his/her conscious reflection of the wider socio-cultural context.

To conclude this description of the process of negotiation of diagnosis, it is important to emphasize that the symptoms of particular disease are objectified within a discourse and then they are applied thus subjectified. It means that after particular disease is assigned, an individual re-interpret his/her health condition. Even though we would refuse the claimed universal "truth" of discourses, we cannot deny that they still ex/implicitly, un/consciously influence person's narratives.

The discussed narration of (not)possessed girl reveals just one side of a coin. It is necessary to analyze other specific example of the influence of discourse on the actual utterance. We can illustrate it on professionals from particular discourses and their questionnaire or specific questions which are derived from the internal logic of the treatment method. These questionnaires and single question - indeed, the discourses on them own - predispose, or even dictate what an individual should recall from his/ her memory. De facto, one's testimony becomes more a reaction then spontaneous revoking of experience. I want to point out that the interviewee is "prodding" to recall some memory from his/her past which is he/she expected to find. Hence, the questions that search for the cause of their condition directly bring implication of their reply. This important aspect can be illustrated on the process of interviewing patient by psychologist and dukun. Opposite to the psychologist's question:: "Have you suffer from any traumatic experience that could affect your condition (negotiated diagnosis)?" we can place dukun's question: "Did you recently visited any dangerous place - cemetery?" In both cases, interviewed person is led by the question to bring out a memory of certain implied moment which is in advance assumed to be the trigger of their undesirable condition. Therefore, asked person will subsequently seek a moment that would correlate with the given question.

Hence, the way of the interpretation and clarification of the disease will be found in a different moments and will be set up in completely different context. Thus the "innocent" and "unbiased" process of questioning, which is trying to uncover the cause of particular disease, significantly affects health seeker's subjective experience and the interpretation of his/her illness. Therefore, the way the illness is asked and told is not just navigating the flow of one's narration, but also formalizing it. Idioms of distress (the way of expressing subjective experiences of illness) therefore do not only form the utterance but determine its content as well.

\section{CONCLUSION}

In this article, I tried to illustrate how discourses shape individual's narratives and change the interpretation and experience of illness. In the discussion of influence of the broader socio-cultural framework and certain institutionalized practices on individual's utterance and experiences, it 
must not be forgotten that this is not a oneway relation. It is important to note the fact that these frameworks / discourses and actors in the social reality are interrelated dialectically (Berger and Luckmann 1967). Structure, objectified and institutionalized practices of diagnosing and the treatment affect the actor, but he/she in turn, creates influences and confirms these various discourses. Therefore it must not be understood as something external to social actors, but as something which is product of their social behavior.

This paper, in which I dealt with variation of individual narrations about their illness and the factors which shape them, I would like to point out that despite interchangeable symptoms, the possession and various mental disorders - these categories must be seen as specific discursive products whose final natures are very different. Therefore the diagnosis, the final constructions of particular discourses, cannot be seen as interchangeable. Hence, for this article it was not important to debate whether one diagnosis or somebodies narrative is "more valid" than the other, or if spirit possession and diagnosis of some mental disorder is a single identical health disorder, or two different. While considering the objective natures of these several diseases we can choose from two standpoints. (1) Possession and a diagnosed mental disorder - schizophrenia or dissociative disorder - or epilepsy can be considered as distinct constructs, which have interchangeable symptoms and are derived from different "objective" phenomena, (2) these constructs are built on one identical phenomenon. However, whether we would incline to any of these variants or not, the conclusion of any discussions about the diagnoses on their own is always the same. The various diseases are different constructs that are due to their definitions are anchored in particular discourses noninterchangeable. In addition it is important to emphasize and reflect that after an individual enter a specific discursive field, his or her illness is remelted into final disease. This assign category and the discourse carve one's self, while the concept of self primarily determi- ne not just prescribed diagnosis but even the discourses. Let us back to poststucturalistic theories. Individual is formed by the social structure and the discourses but these structures are on the other hand created by the social actors.

Compared to my conclusion which highlights differences between narrations and diagnosis of spirit possession and mental disorders which are contingent on the reference framework, their final natures are discursively determined - in the context of the Czech Republic Konopásek and Palecek discuss "interdeffiusion" of the phenomenon of appearance of spiritual entity and certain mental disorders (2012). However, it is important to emphasize what they mentioned only marginally. Reflecting Sudnow (1967) it is important to underline once again that the particular diagnosis is an interpretation which objectifies individual condition and this is consequently linked with an institutionalized practice that is assumed to treat the assigned disease.

Therefore, it is obvious that although we would assume in theoretical discussion, that there is an objective phenomenon which might be understood as border one, we can assume that only about the phenomenon before it is carved by various discourses into particular diseases, which are discursively constituted and anchored in the framework of the relevant meaning. That is because the fact that after the diagnosis are given, these construct are firmly noninterchangeable.

In addition it is important to emphasize that narrations which are linked with the selection of chosen treatment of spirit possession and/or a mental disorder in West Sumatra meet the same characteristics and trajectories of the treatment seeking as described by Kurihara et al (2006). Affected individual first searches help from the closest social surrounding. This primarily consists of the revision of social relationships and requirements. If this is not successful, the individual who assume or is assumed to be spirit possessed goes or is sent first to ruqyah or dukun. Only as the last possibility individual seeks help from psychiatrists or psychologists. 


\section{REFERENCES}

Acharya, M. P. and Northcott, H. C. 2007. Mental Distress and the Coping Strategies of

Elderly Indian Immigrant Women. Transcultural Psychiatry. 44: 614-367.

Barnard, A. \& Spencer, J. 1996. Encyclopedia of Social and Cultural Antropology. New York: Routledge.

Berger P. and Luckmann T. 1967. The Social Construction of Reality: A Treatise in the Sociology of Knowledge. New York: Anchor Books.

Bourdieu P. 1998. Practical Reason: On the Theory of Action. Stanford University Press.

Csordas, T.J., et al. 2010. Ways of Asking, Ways of Telling. Culture, Medicine, and Psychiatry, 34(1):29-55.

Foucault, M. 1994. Discipline and Punish: The Birth of the Prison. London: Penguin Books.

Good, B. 1977. The Heart of What's the Matter: The Semantics of Illness in Iran. Culture, Medicine and Psychiatry. April (1): 25-58.

Helman, C. 1978. Feed a Cold, Starve a Fever: Folk Models of Infection in an English Suburban Community and Their Relations to Medical Treatment. Culture, Medicine andPsychiatry. 2: 107-137.

Hollan, D. 2004. Self Systems, Cultural Idioms of Distress, and the Psycho-Bodily Consequences of Childhood Suffering. Transcultural Psychiatry. 41: 62-79.
Kirmayer, L. J. 2006. Beyond the 'New Cross-cultural Psychiatry: Cultural Biology, Discursive Psychology and the Ironies of Globalization. Transcultural Psychiatry. 43 (1): 126-144.

Kohrt, B. A. and Hruschka D. J. 2010. Nepali Concepts of Psychological Trauma: The Role of Idioms of Distress, Ethnopsychology and Ethnophysiology in Alleviating Suffering and Preventing Stigma. Culture, Medicine and Psychiatry. 34: 322-352.

Konopásek, Z. and Paleček, J. 2012. Apparitions and Possessions as Boundary Objects: An Exploration into Some Tensions between Mental Health Care and Pastoral Care. Journal of Religion and Health. 51 (3): 970-985.

Kurihara, T., Kato, M., Reverger, R. Rai Tirta, G. 2006. Pathway to psychiatric care in Bali. Psychiatry and Clinical Neurosciences. 60: 204-210.

Latour, B. 1999. Thou shalt not take the Lord's name in vain - being a sort of sermon. Sociology of Religion. 39: 215-234.

Littlewood, R. 1996. Psychiatry's Culture. International Journal of Social Psychiatry. 42: 245-268.

Von Peter, S. 2008. The Experience of 'Mental Trauma' and its Transcultural Application. Transcultural Psychiatry. 45: 639-651.

Práško J., et al. 2007. Disociativní poruchy a jejich léčba. Medicína pro Praxi. 4 (9): 471-476.

Sudnow D. 1967. Passing on, The social Organization of Dying. New Jersey: Prentice-Hall 